\title{
Identifizierung von Lysin- und Argininresten als Hemmzentren von Proteaseinhibitoren mit Hilfe von Maleinsäure- anhydrid und Butandion-(2.3)
}

\author{
Von Hans fritz*, Edwin Fink, Maria Gebhardt, Karl Hochstrasser und Eugen Werle \\ Aus dem Institut für Klinische Chemie und Klinische Biochemie der Universität München
}

(Der Schriftleitung zugegangen am 23. Mai 1969)

\begin{abstract}
Zusammenfassung: Die folgenden Inhibitoren haben nach Umsetzung mit Maleinsäureanhydrid ihre Hemmwirkung gegenüber den angegebenen Enzymen verloren: Inhibitor aus Pankreas von Schwein, Hund und Katze (Trypsin); Meerschweinchen-Samenblasen II, Hirudin und Limabohnen (Trypsin und Plasmin); Lunge von Rind und Schaf (Trypsin, Plasmin, Kallikrein und Chymotrypsin). Die antichymotryptische Aktivität des Inhibitors aus Limabohnen wird durch die Acylierung der Aminogruppen nicht beeinflußt. Die genannten Inhibitoren werden in saurer Lösung deacyliert, wobei sie ihre Aktivität zurückgewinnen. Die Polymaleoyl-Derivate der Inhibitoren aus Weißbohnen und Meerschweinchen-Samenblasen besitzen noch etwa $1 / 3$ der antitryptischen Aktivität der nativen Inhibitoren. Der Verlust der Hemmwirkung gegenüber Trypsin bzw. Plasmin und Kallikrein nach der Umsetzung mit Maleinsäureanhydrid wird auf die Acylierung der Aminogruppe eines Lysinrestes zurückgeführt, der sich im reaktiven Zentrum des Inhibitors befindet.
\end{abstract}

Summary: Identification of lysine and arginine residues as reactive centres of protease inhibitors with
Einen Argininrest im reaktiven Zentrum besitzen die folgenden Inhibitoren: Inhibitor aus Schafspankreas, Gl. submand. vom Hund, Sojabohnen, Hühnereiklar, Weizenkeimen, Roggenkeimen, Kartoffeln, Erdnüssen sowie der Inter- $\alpha$-Trypsin-Inhibitor aus Humanserum. Die Polymaleoyl-Derivate dieser Inhibitoren, die dieselbe antitryptische Wirkung wie die nativen Inhibitoren besitzen, werden bei der Umsetzung mit einem Butandion(2.3)-Reagens relativ rasch und irreversibel inaktiviert. Dieses Reagens modifiziert nach der Acylierung der Aminogruppen der Inhibitoren spezifisch die Guanidinogruppen der Argininreste. Die Antiplasminaktivität der Inhibitoren aus Gl. submand. vom Hund, Sojabohnen und Erdnüssen ist nach Acylierung der Aminogruppen ebenfalls nicht vermindert; sie nimmt jedoch bei der Einwirkung des Butandion-(2.3)-Reagens auf die PolymaleoylDerivate dieser Inhibitoren parallel zur Antitrypsinaktivität ab.

\footnotetext{
* Postanschrift: Dr. HaNs FrITZ, D-8 München 15, Nußbaumstr. 20.

Abkürzungen:

BAEE $=N^{\alpha}$-Benzoyl-L-arginin-äthylester; BAPNA $=N^{\alpha}$-Benzoyl-DL-arginin-p-nitro-anilid;

CPPN $=N$-[3-Carboxy-propionyl]-L-phenylalanin-p-nitro-anilid.

Enzyme:

Carboxypeptidase B, Peptidyl-L-Lysin-Hydrolase (EC 3.4.2.2)

Chymotrypsin A (EC 3.4.4.5)

Kallikrein (EC 3.4.4.21)

Plasmin (EC 3.4.4.14)

Trypsin (EC 3.4.4.4)
}

the aid of maleic anhydride and 2,3-butanedione. The following inhibitors were treated with maleic an- 
hydride, whereupon they lost their inhibitory acivity towards the given enzymes: inhibitor from swine, dog and cat pancreas (trypsin); from guinea pig seminal vesicles II, hirudin and lima beans (trypsin and plasmin); from bovine and sheep lung (trypsin, plasmin, kallikrein and chymotrypsin). The antichymotryptic activity of the inhibitor from lima beans was not affected by acylation of the amino groups. The inhibitors regained their inhibitory activity after deacylation in acidic solution. The polymaleoyl derivatives of the inhibitors from haricot beans and guinea pig seminal vesicles still possessed about one third of the antitryptic activity of the native inhibitors. The loss of inhibition towards trypsin or plasmin and kallikrein after reaction with maleic anhydride is due to the acylation of the amino group of a lysine residue, which is in the reactive centre of the inhibitor.

The following inhibitors contain an arginine resi- due in the reactive centre: inhibitor from sheep pancreas, from Gl. submandibularis of the dog, from soya bean, from hen egg white, wheat shoots, rye shoots, potatoes, ground nuts, and the inter- $\alpha-$ trypsin inhibitor from human serum. The polymaleoyl derivatives of these inhibitors, which possess the same antitryptic activity as the native inhibitors, are inactivated irreversibly and relatively quickly by reaction with a 2,3-butanedione reagent. This reagent modifies specifically the guanidino groups of the arginine residues after acylation of the amino groups of the inhibitors. The antiplasmin activity of the inhibitors from Gl. submandib. of the dog, soya bean and ground nuts is not decreased after the acylation of the amino groups; but when the polymaleoyl derivatives of these inhibitors are treated with 2,3-butanedione reagent, the decrease of their antiplasmin activities parallels that of their antitrypsin activities.
Das ,aktive Zentrum“ oder Hemmzentrum von Trypsininhibitoren stellt nach neueren Untersuchungen ein Lysin- oder Argininrest bzw. eine Peptidsequenz dar ${ }^{1-7}$, für die Trypsin eine besonders hohe Affinität besitzt. Wir untersuchten deshalb den Einfluß der Substitution von $\varepsilon$-Aminogruppen von Lysylresten und der Modifizierung der Guanidinogruppen der Arginylreste auf die Inhibierungsfähigkeit einer größeren Zahl von Proteaseinhibitoren. Zur reversiblen Substitution von Aminogruppen von Proteinen eignet sich Maleinsäureanhydrid ${ }^{8}$; nach Acylierung der Aminogruppen sind dann mit Hilfe eines Butandion-(2.3)Reagens spezifisch die Argininreste der Proteine

1 W. R. Finkenstadt u. M. Laskowski JR., J. biol. Chemistry 240, PC 962 [1965].

2 Dieselben, J. biol. Chemistry 242, 771 [1967].

3 K. Ozawa u. M. Laskowski JR., J. biol. Chemistry 241, 3955 [1966].

4 R. Haynes u. R. E. Feeney, J. biol. Chemistry 242 , 5378 [1967].

5 R. Haynes, D. T. Osuga u. R. E. Feeney, Biochemistry [Washington] 6, 541 [1967].

${ }^{6}$ H.-H. Liu, G. Feinstein, D. T. Osuga, R. Haynes u. R. E. FeENeY, Biochemistry [Washington] 7, 2886 [1968].

7 R. Haynes u. R. E. Feeney, Biochemistry [Washington] 7, 2879 [1968].

8 P. J. G. Butler, J. I. Harris, B. S. Hartley u. R. Leberman, Biochem. J. 103, 78 P [1967]; 112, 679 [1969]. modifizierbar ${ }^{9}$. Bei polyvalenten Proteaseinhibitoren wurde der Einfluß der Substitution und Modifizierung auch auf die Hemmwirkung gegenüber den weiteren in Frage kommenden Enzymen (Chymotrypsin, Plasmin, Kallikreine) untersucht.

\section{Material und Methoden}

\section{Material}

Enzyme: Trypsin, $\alpha$-Chymotrypsin und Plasmin, Novo Industrie; Kallikrein aus Schweinepankreas nach FrITZ u. Mitarb. ${ }^{10}$. Inhibitoren: vgl. Tab. 1. Reagentien: Maleinsäureanhydrid (Furandion-(2.5), Schuchardt; Butandion-(2.3), EGA-Chemie; Azocasein, Fluka.

\section{Methoden}

1. Bestimmung der Aktivität und Hemmung der Enzyme

Trypsin

a) Substrat $N^{\alpha}$-Benzoyl-DL-arginin-p-nitro-anilid (BAPNA) ${ }^{11,12}$; 1 IU entspricht dabei der Hemmung der Aktivität von ca. $1 \mathrm{mg}$ Trypsin Novo.

9 A. L. Grossberg u. D. Pressman, Biochemistry [Washington] 7, 272 [1968].

10 H. Fritz, I. Eckert u. E. Werle, diese Z. 348, 1120 [1967].

11 H. Fritz, G. Hartwich u. E. Werle, diese Z. 345, 150 [1966].

12 H. Fritz, I. Trautschold u. E. Werle, in H. U. BergmeYer, Methoden d. enzymat. Analyse, Verlag Chemie, im Druck. 
b) Substrat Azocasein ${ }^{12}$; Durchführung: $1 \mathrm{ml}$ Phosphatpuffer, $\mathrm{pH} 7,6$, enthaltend 1-4 $\mu \mathrm{g}$ Trypsin (Bezugsansatz) bzw. $4 \mu \mathrm{g}$ Trypsin plus entsprechende Inhibitormenge (Hemmansatz, 1-5 Min. vorinkubieren!) wird mit $2,0 \mathrm{ml} 2$ proz. Azocaseinlösung (in Phosphatpuffer) versetzt, die Mischung $10 \mathrm{Min}$. bei $30^{\circ} \mathrm{C}$ inkubiert, dann durch Zugabe von 3,0 $\mathrm{ml} 5$ proz. Trichloressigsäure das nicht umgesetzte Substrat gefällt, nach 30 Min. langem Stehenlassen bei Zimmertemperatur zentrifugiert und die Extinktion der überstehenden Lösung bei $366 \mathrm{~nm}$ gegen den Reagentienleerwert gemessen. Zwischen $E=0,065$ (ca. $1 \mu \mathrm{g}$ Trypsin) und $E=$ 0,250 (ca. $4 \mu \mathrm{g}$ Trypsin) nimmt die Extinktion des Bezugsansatzes proportional zur Trypsinkonzentration zu. Die verminderte Extinktion des Hemmansatzes gegenüber der des Bezugsansatzes dient als $\mathrm{Maß}$ für den Inhibitorgehalt (in $\mu \mathrm{g}$ Trypsin gehemmt) des Hemmansatzes bzw. der zugegebenen Probe.

\section{Chymotrypsin}

Substrat: $\quad N$-[3-Carboxy-propionyl]-L-phenylalanin-pnitro-anilid (CPPN) ${ }^{\mathbf{1 2}, 13}$. Die Inhibitoraktivität ist durchwegs angegeben in $\mathrm{mg}$ gehemmtes Chymotrypsin/mg Inhibitor.

\section{Plasmin}

a) Substrat: BAPNA ${ }^{12}$. 1 Novo-Einheit Plasmin entspricht im BAPNA-Test (0,05M L-Lysin statt Calciumchlorid im Puffer) etwa 4,2 mU, d.h. einer Extinktionsänderung $\Delta E_{405}=0,140$ in $10 \mathrm{Min}$. Meßbereich: 0,8-8,4 mU (entspricht ca. 0,2 bis 2 Novo-Einheiten Plasmin). 1 IU für Plasmin entspricht somit der Hemmung von ca. 0,25 Novo-Einheiten Plasmin.

b) Substrat: Azocasein ${ }^{12}$. Durchführung wie beim Trypsin angegeben. Meßbereich: $E_{366}=0,100$ bis $E_{366}=0,700$ (entsprechend ca. 0,3 bis 2,0 Novo-Einheiten Plasmin).

\section{Kallikrein}

a) Substrat: $N^{\alpha}$-Benzoyl-L-arginin-äthylester (BAEE) 10,12 .

b) Vergleichende Blutdrucksenkung beim Hund ${ }^{14}$. $1 \mathrm{U}$ bei der BAEE-Spaltung entspricht ca. 10 biologischen Kallikrein-Einheiten.

13 H. Fritz, F. Woitinas u. E. Werle, diese Z. 345, 168 [1966].

14 E. K. Frey, H. Kraut u. E. Werle, Das KallikreinKinin-System und seine Inhibitoren, Stuttgart, EnkeVerlag 1968.

15 H. Fritz, I. Hüller, M. Wiedemann u. E. Werle, diese Z. 348, 405 [1967]; H. FrITZ, H. Schult, M. HUTZel, M. WiedemanN u. E. Werle, diese Z. 348, 308 [1967].

16 H. Fritz, M. Gebhardt, E. Fink, W. Schramm u. E. Werle, diese Z. 350, 129 [1969].

17 H. Fritz, E. Fink, R. Meister u. E. Werle, in Vorbereitung.
Tab. 1. Trypsininhibitoren: Hersteller- und Bezugsquellennachweis. Der Reinheitsgrad der verwendeten Präparate ist aus Tab. 2 zu ersehen.

\begin{tabular}{ll}
\hline Trypsininhibitor aus & $\begin{array}{l}\text { Hergestellt nach } \\
\text { bzw. Bezugsquelle }\end{array}$ \\
\hline $\begin{array}{l}\text { Pankreas von } \\
\text { Schwein }\end{array}$ & FrITZ u. Mitarb. ${ }^{15}$ \\
Hund & FRITZ u. Mitarb. ${ }^{15}$ \\
Katze & FRITZ u. Mitarb. ${ }^{15,16}$ \\
Schaf & FRITZ u. Mitarb. ${ }^{16}$
\end{tabular}

Meerschweinchen-Samen-

blasen

I: Trypsin-Inhibitor FrITZ u. Mitarb. 16,17

II: Trypsin-Plasmin-Inh. FrITZ u. Mitarb.16,17

Gl. Submand. (Hund) FRITZ u. Mitarb. ${ }^{18}$

Lunge von

Rind

Schaf

Hirudin ,Bdellin“

„Trasylol,,, Farbenfabriken

Bayer, Werk Elberfeld

Weizenkeime I

(Triticum vulgare)

FRITZ u. Mitarb.16,19

Roggenkeime I

(Secale cereale)

FrITz u. Mitarb. ${ }^{20}$

Weißbohnen

(Phaseolus vulgaris) HochSTRASSER u. Mitarb. ${ }^{22}$

Kartoffeln I

(Solanum tuberosum) HochSTRASSER u. Mitarb. ${ }^{23}$

Erdnuß PNT

(Arachis hypogaea) HochSTRASSER u. Mitarb. ${ }^{24}$

Sojabohnen (Glycine soja) pract. lyophil, Serva Heidel-

Eiklar (Huhn)

„Ovomucoid“ Worthington, Freehold

Limabohnen

Inter- $\alpha$-Trypsin-Inhibitor (Humanserum)

M 2113, Mann Research, N.Y.

Geschenk der Behringwerke, Marburg

18 H. Fritz, P. Pasquay u. E. Werle, in Vorbereitung. 19 H. Fritz, W. Schramm, B. Greif u. E. Werle, in Vorbereitung.

20 H. FrITZ, K.-H. OpPITZ, M. GebhaRdT, I. OpPITZ u. E. WERLE, diese Z. 350, 91 [1969].

${ }^{21}$ K. Hochstrasser u. E. Werle, diese Z. 350, 249 [1969].

22 K. Hochstrasser u. E. Werle, diese Z., in Vorbereitg.

23 K. Hochstrasser, E. Werle, R. SiegelmanN u. S. SCHWARZ, diese Z. 350, 897 [1969].

24 K. Hochstrasser, K. IllchmanN u. E. Werle, diese Z. 350, 929 [1969], vorstehend. 


\section{Umsetzung der Inhibitoren mit Maleinsäureanhydrid}

a) $1 \mathrm{mg}$ Inhibitor, $15 \mathrm{mg}$ Natriumhydrogencarbonat und $10 \mathrm{mg}$ gepulvertes Maleinsäureanhydrid wurden mit einem Glasstab in einem Zentrifugenglas verrieben und zur Mischung $0,25 \mathrm{ml} 0,1 \mathrm{M} \mathrm{NaHCO}_{3}$-Lösung gegeben. Die Suspension wurde unter Eiskühlung $\left(0-4^{\circ} \mathrm{C}\right)$ geschüttelt, bis eine klare Lösung entstand (ca. 10 Min.). Danach wurden mit $0,25 \mathrm{ml}$ dest. Wasser am Glasrand haftende Feststoffteilchen in den Reaktionsansatz gespült und dieser wurde weitere $30 \mathrm{Min}$. bei Zimmertemperatur unter gelegentlichem Schütteln stehengelassen. Zur Bestimmung der Inhibitoraktivität in der Reaktionslösung wurden geeignete Verdünnungen davon zum jeweiligen Hemmtest eingesetzt. Die spezifische Aktivität des Ausgangspräparates wurde mittels einer gesonderten Einwaage bestimmt.

b) Die Lösung von $1 \mathrm{mg}$ des Inhibitors in $0,5 \mathrm{ml} 0,5 \mathrm{M}$ $\mathrm{NaHCO}_{3}$-Lösung wurde mit $10 \mathrm{mg}$ gepulvertem Maleinsäureanhydrid versetzt, die Mischung unter Eiskühlung $\left(0-4^{\circ} \mathrm{C}\right) 10 \mathrm{Min}$. geschüttelt und danach $30 \mathrm{Min}$. bei Zimmertemperatur unter gelegentlichem Schütteln stehengelassen. Danach wurde mit Hilfe geeigneter Verdünnungen die Inhibitoraktivität in der Reaktionslösung bestimmt; die Inhibitoraktivität der Ausgangslösung wurde vor Zugabe des Maleinsäureanhydrids getestet.

\section{Freisetzung der Inhibitoren aus ihren Polymaleoyl- Derivaten}

$0,1 \mathrm{ml}$ des Reaktionsansatzes $2 \mathrm{a}$ ) oder $2 \mathrm{~b}$ ) wurden mit $0,9 \mathrm{ml} 3$ proz. Perchlorsäure versetzt und die saure Lösung bei $60^{\circ} \mathrm{C}$ inkubiert. $\mathrm{Zu}$ den in Tab. 3 angegebenen Zeitpunkten wurden Proben entnommen und ihre Inhibitoraktivität bestimmt.

\section{Umsetzung der Polymaleoyl-Inhibitoren mit Butan- dion-(2.3)-Reagens}

Die Herstellung des Butandion-(2.3)-Reagens erfolgte nach den Angaben von Grossberg und Pressman ${ }^{9}$ : Die Extinktion einer 15 fachen Verdünnung des Reagens betrug nach 14stdg. Stehenlassen bei Zimmertemperatur bei $405 \mathrm{~nm} \mathrm{0,65}$.

$0,2 \mathrm{ml}$ der Lösung des Polymaleoyl-Inhibitors (Reaktionsansatz von $2 \mathrm{a}$ ) oder $2 \mathrm{~b}$ )) wurden mit $0,2 \mathrm{ml} 1 \mathrm{M}$ $\mathrm{NaHCO}_{3} / \mathrm{Na}_{2} \mathrm{CO}_{3}$-Puffer, pH 8,6, und mit $0,2 \mathrm{ml}$ des Butandion-2.3-Reagens versetzt. Die gut vermischte Reaktionslösung wurde danach bei Zimmertemperatur inkubiert. $\mathrm{Zu}$ den in Tab. 5 angegebenen Zeitpunkten wurden Proben entnommen und nach entsprechender Verdünnung wurde ihre Inhibitoraktivität bestimmt. Die beim Test vorgelegte Trypsinaktivität wird durch das Butandion-(2.3)-Reagens nicht beeinflußt, wie ein nach Zugabe von $0,2 \mathrm{~m} l$ Reagens gemessener Vergleichswert ergab.

\section{Ergebnisse}

\section{Beeinflussung des Hemmverhaltens gegenüber Trypsin}

Aufgrund des Hemmverhaltens der Proteaseinhibitoren gegenüber Trypsin (Substrat: BAPNA) nach der Reaktion mit Maleinsäureanhydrid lassen sich 3 Gruppen von Inhibitoren unterscheiden:

1. Die sog. Lysin-Inhibitoren (Tab. 2) haben nach Substitution der Aminogruppen ihre Hemmwirkung gegenüber Trypsin verloren. Die verbleibende Restaktivität von 3,4 bis weniger als $0,1 \%$ der eingesetzten Inhibitoraktivität wird durch $\mathrm{Zu}$ gabe eines noch größeren Uberschusses an Maleinsäureanhydrid weiter vermindert, ist also auf die unter den angewandten Bedingungen nicht quantitativ verlaufene Umsetzung zurückzuführen.

Durch Säureeinwirkung werden die Lysin-Inhibitoren aus ihren inaktiven Polymaleoyl-Derivaten wieder freigesetzt (Tab. 2,3 und Abb. 1). Die Hemmaktivität gegenüber Trypsin erreicht bei den säurestabilen Inhibitoren im allgemeinen nach 2stdg. Inkubation in 3proz. Perchlorsäure wieder den ursprünglichen Wert $(100-112 \%)$. Geringere Ausbeuten bei der Reaktivierung (67 und $75 \%$ in Tab. 3, vgl. Fußnote b) sind auf eine Zerstörung (hydrolytische Spaltung) der betreffenden Inhibitoren in der stark sauren Lösung zurückzuführen.

2. Bei den sog. Arginin-Inhibitoren (Tab. 4) bleibt nach der Umsetzung mit Maleinsäureanhydrid die Hemmwirkung gegenüber Trypsin vollständig erhalten. Eine geringe Zunahme der Inhibitoraktivität (Inhibitor aus Sojabohnen und Erdnuß) ist evt. auf die Dissoziation von Polymeren zurückzuführen, eine geringe Abnahme (Inhibitor aus Kartoffeln I und Inter- $\alpha$-Trypsin-Inhibitor) evt. auf eine teilweise Denaturierung bei der Umsetzung infolge des relativ hohen Salzgehaltes der Lösungen.

Die Polymaleoyl-Derivate der Arginin-Inhibitoren verlieren dagegen bei der Umsetzung mit dem Butandion-(2.3)-Reagens relativ rasch ihre Hemmwirkung gegenüber Trypsin (Tab. 4 und 5): Die verbleibende Restaktivität beträgt nach 1,5-2stdg. Inkubation in allen Fällen weniger als $10 \%$ des Ausgangswertes und nimmt bei längerer Inkubation noch weiter $a b$.

3. Ein von den Lysin- bzw. Arginin-Inhibitoren abweichendes Verhalten zeigen die Inhibitoren aus Meerschweinchen-Samenblasen I und Weißboh- 
Tab. 2. Lysin-Inhibitoren. Verbleibende Aktivität nach Umsetzung mit Maleinsäureanhydrid und nach Säurebehandlung der inaktiven Polymaleoyl-Derivate.

Die Versuchsdurchführung ist im Abschnitt Methodik beschrieben. Für die Bestimmung der Hemmung der Trypsin- und Plasminaktivität wurde, sofern nicht anders angegeben, das Substrat BAPNA verwendet. - Vor der Umsetzung mit Maleinsäureanhydrid hemmte $1 \mathrm{mg}$ des Inhibitors aus Rinderlunge die Aktivität von 3,0 mg Chymotrypsin sowie 1600 biologische Kallikrein-Einheiten (entsprechend einer Hemmung von $160 \mathrm{U}$ bei der BAEE-Spaltung); $1 \mathrm{mg}$ des Inhibitors der Schafslunge hemmte die Aktivität von 3,2 mg Chymotrypsin und 2140 biologische Kallikrein-Einheiten.

\begin{tabular}{|c|c|c|c|c|c|c|c|c|c|}
\hline \multirow{2}{*}{\multicolumn{2}{|c|}{ Trypsininhibitor aus }} & & \multicolumn{2}{|c|}{$\begin{array}{l}\text { Spezif. Aktiv. } \\
\text { [IU/mg] }\end{array}$} & \multicolumn{4}{|c|}{$\begin{array}{c}\text { Verbleibende Aktiv. in } \% \mathbf{a} \\
\text { n. Umsetzg. mit } \\
\text { Maleinsäureanhydrid }\end{array}$} & \multirow{2}{*}{$\begin{array}{c}\text { Freigesetzte Aktivität } \\
\text { in \% \% nach } 2 \text { stdg. } \\
\text { Säureeinwirkung } \\
\text { Trypsin }\end{array}$} \\
\hline & & & Trypsin & n Plasmin & Trypsin & Plasmin & Chymotr. & Kallikr. & \\
\hline $\begin{array}{l}\text { Pankreas } \\
\text { von }\end{array}\{$ & $\left\{\begin{array}{l}\text { Schwein } \\
\text { Hund } \\
\text { Katze }\end{array}\right.$ & & $\begin{array}{l}2,9 \\
3,7 \\
3,6\end{array}$ & & $\begin{array}{r}0,2 \\
<0,1 \\
0,8\end{array}$ & & & & $\begin{array}{c}110 \\
78^{b} \\
112\end{array}$ \\
\hline \multicolumn{2}{|c|}{$\begin{array}{l}\text { Meerschweinchen- } \\
\text { samenblasen }\end{array}$} & $\begin{array}{r}\text { I } \\
\text { II }\end{array}$ & $\begin{array}{l}3,9 \\
4,7\end{array}$ & (3, 0,25 & $\begin{array}{l}32 \\
2,2\end{array}$ & 3,3 & & & $\begin{array}{r}100^{d} \\
67^{b}\end{array}$ \\
\hline $\begin{array}{l}\text { Lunge } \\
\text { von }\end{array}$ & $\left\{\begin{array}{l}\text { Rind } \\
\text { Schaf }\end{array}\right.$ & & $\begin{array}{l}3,0 \\
3,3\end{array}$ & $\begin{array}{r}120^{c} \\
0,26\end{array}$ & $\begin{array}{l}0,6 \\
2,5\end{array}$ & $\begin{array}{l}1,7 \\
2,7\end{array}$ & $\begin{array}{l}1,2 \\
0,5\end{array}$ & $\begin{array}{l}1,0 \\
1,6\end{array}$ & $\begin{array}{l}100 \\
104\end{array}$ \\
\hline \multicolumn{3}{|c|}{ Hirudin „Bdellin““ } & 3,5 & 0,29 & 3,4 & 2,5 & & & 86 \\
\hline \multicolumn{3}{|c|}{ Limabohnen } & 3,2 & 0,09 & 1,9 & 1,9 & $112^{\mathrm{e}}$ & & $67^{\mathrm{b}}$ \\
\hline \multicolumn{2}{|c|}{ Weißbohnen } & & 4,5 & & 33 & & & & \\
\hline \multicolumn{6}{|c|}{$\begin{array}{l}\text { Bezogen auf die zur Umsetzung mit Maleinsäureanhydrid ein- } \\
\text { gesetzte Aktivität. } \\
\text { b Diese Inhibitoren werden unter den angewandten Bedingungen } \\
\text { langsam zerstört; vgl. Tab. } 3 \text {. } \\
\text { c BAEE als Substrat. }\end{array}$} & $\begin{array}{l}\text { d Nach } 20 \\
\text { e Vor Ur } \\
\text { Inhibito } \\
\text { setzung } \\
\text { gens wu }\end{array}$ & $\begin{array}{l}\text { stdg. Säureei } \\
\text { nsetzung mi } \\
\text { rs die Aktivi } \\
\text { des Polymale } \\
\text { rde die Aktiv }\end{array}$ & $\begin{array}{l}\text { irkung. } \\
\text { Maleinsäu } \\
\text { von } 0,49 \\
\text {-Derivate } \\
\text { t von } 0,61\end{array}$ & $\begin{array}{l}\text { hydrid hemmte } 1 \mathrm{mg} \text { des } \\
\text { Chymotrypsin; nach Um- } \\
\text { it dem Butandion-(2.3)-Rea- } \\
\text { Chymotrypsin gehemmt. }\end{array}$ \\
\hline
\end{tabular}

Tab. 3. Lysin-Inhibitoren. Freisetzung der Trypsininhibitoren aus ihren Polymaleoyl-Derivaten.

Die Reaktionsbedingungen sind im Abschnitt Methodik beschrieben. Die in Spalte 2 angegebenen Mengen sind aus den bei der Herstellung der Polymaleoyl-Derivate eingesetzten Inhibitoraktivitäten berechnet.

\begin{tabular}{|c|c|c|c|c|c|c|c|c|}
\hline \multirow{2}{*}{\multicolumn{2}{|c|}{$\begin{array}{l}\text { Polymaleoyl-Derivat } \\
\text { des Inhibitors aus }\end{array}$}} & \multirow[t]{2}{*}{$\begin{array}{l}\text { Eingesetzte Menge } \\
{[\mathrm{mIU}]}\end{array}$} & \multicolumn{6}{|c|}{$\begin{array}{l}\text { Freigesetzte Aktivität (in } \% \text { ) in saurer Lösung nach } \\
\text { verschieden langer Inkubationszeit (in Min.) }\end{array}$} \\
\hline & & & 10 & 25 & 30 & 60 & 120 & 1200 \\
\hline $\begin{array}{l}\text { Pankreas } \\
\text { von }\end{array}$ & $\begin{array}{l}\text { Schwein } \\
\text { Hund } \\
\text { Katze }\end{array}$ & $\begin{array}{l}575 \\
740 \\
730\end{array}$ & $\begin{array}{r}13 \\
9 \\
12\end{array}$ & & & $\begin{array}{l}52 \\
53 \\
62\end{array}$ & $\begin{array}{c}110 \\
75^{b} \\
112\end{array}$ & 49 \\
\hline $\begin{array}{l}\text { Meerschw } \\
\text { Samenblas }\end{array}$ & $\begin{array}{lr}\text { einchen- } & \text { I } \\
\text { sen } & \text { II }\end{array}$ & $\begin{array}{l}784^{c} \\
934\end{array}$ & & & $\begin{array}{l}46 \\
65\end{array}$ & & $\begin{array}{l}54 \\
67 \mathrm{~b}\end{array}$ & 100 \\
\hline $\begin{array}{l}\text { Lunge } \\
\text { von }\end{array}$ & $\begin{array}{l}\text { Rind } \\
\text { Schaf }\end{array}$ & $\begin{array}{l}603 \\
665\end{array}$ & 19 & & & 75 & $\begin{array}{l}100 \\
104\end{array}$ & \\
\hline Hirudin , & Bdellin“" & 700 & 16 & 35 & & 52 & 86 & \\
\hline Limabohn & & 632 & & & 67 & & $67^{b}$ & $<1$ \\
\hline
\end{tabular}

a Bezogen auf die zur Umsetzung mit Maleinsäureanhydrid eingesetzte Aktivität (Tab. 2).

b Diese Inhibitoren werden unter den angewandten Bedingungen langsam inaktiviert: Gefundener Aktivitätsverlust der nicht modi- fizierten Inhibitoren in 3proz. Perchlorsäure bei $60^{\circ} \mathrm{C}$ nach 150 Min.: $13-14 \%$.

c Davon 211 mIU frei, d. h. testbar (vgl. Tab. 2). 


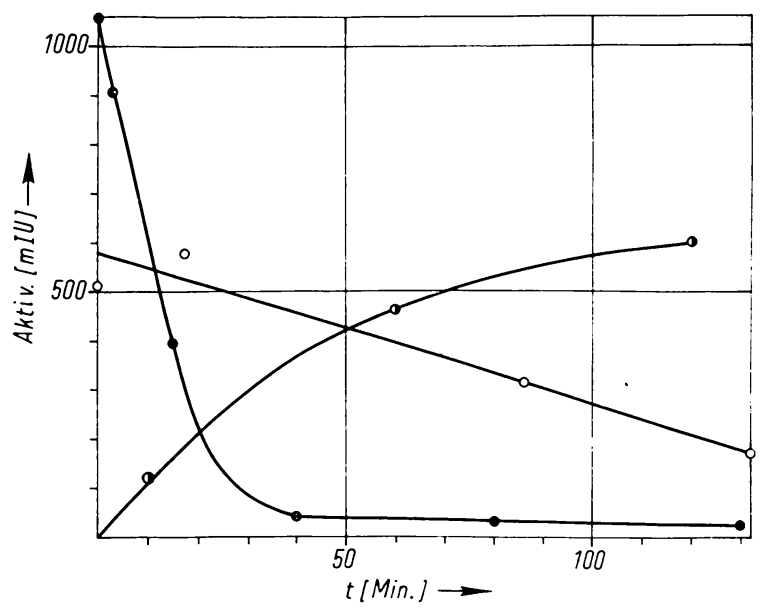

Abb. 1. Inaktivierungsgeschwindigkeiten der Polymaleo-

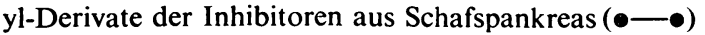
und aus Weißbohnen $(\circ-\infty)$ bei Einwirkung des Butandion-(2.3)-Reagens sowie Freisetzungsgeschwindigkeit des Inhibitors aus Rinderlunge ( $-\infty$ ) aus dem Polymaleoyl-Derivat.

Die Versuchsdurchführung ist im Abschnitt Methodik beschrieben. Vgl. dazu Tab. 5 und 3.

nen: Beide Inhibitoren verlieren bei der Umsetzung mit Maleinsäureanhydrid nur etwa $2 / 3$ ihrer Hemmwirkung gegenüber Trypsin, gemessen mit dem synthetischen Substrat BAPNA (Tab. 2 und 4); mit Azocasein als Substrat findet man einen Aktivitätsverlust von derselben Größenordnung. Die Restaktivität von $33 \%$ beim Polymaleoyl-Derivat des Inhibitors aus Weißbohnen (Tab. 2 und 4) nimmt bei der Reaktion mit dem Butandion-(2.3)Reagens wesentlich langsamer ab als die der Polymaleoyl-Derivate der Arginin-Inhibitoren (Tab. 5 und $A b b .1$ ). Dies spricht dafür ${ }^{9}$, daß für die verbleibende Hemmaktivität dieses Inhibitors eine Aminogruppe und nicht eine Guanidinogruppe verantwortlich ist. Demnach ist der Inhibitor aus Weißbohnen der Gruppe der Lysin-Inhibitoren zuzuordnen, wobei allerdings ein von den anderen Inhibitoren sich unterscheidender Hemmechanismus zu diskutieren ist. Letzteres gilt auch für den Inhibitor I aus Meerschweinchen-Samenblasen, bei dem die im Polymaleoyl-Derivat noch vorhandene Restaktivität bei der Einwirkung des Butandion-(2.3)-Reagens ebenso rasch wie bei den Arginin-Inhibitoren verschwindet (Tab. 5). Beim Polymaleoyl-Derivat des Inhibitors I aus Meerschweinchen-Samenblasen ist außerdem die zwar relativ langsam verlaufende aber völlige Reaktivierung in saurer Lösung bemerkenswert.

\section{Beeinflussung des Hemmverhaltens polyvalenter Inhibitoren}

Das Hemmverhalten der folgenden Lysin-Inhibitoren veränderte sich bei der Umsetzung mit Maleinsäureanhydrid gleichsinnig (Tab. 2): Inhibitoren aus Lunge von Rind und Schaf, Inhibitor II aus Meerschweinchen-Samenblasen und Inhibitor „Bdellin“ aus Hirudin. Diese Inhibitoren verlieren durch die Substitution der Aminogruppen nicht nur ihre Hemmwirkung gegenüber Trypsin sondern - innerhalb der methodischen Fehlergrenzen - in demselben Maße auch gegenüber Plasmin, Chymotrypsin und Kallikrein. Derselbe Befund trifft auch für die Plasmin-hemmende Wirkung des Inhibitors aus Limabohnen zu, während die antichymotryptische Aktivität dieses Inhibitors durch die Substitution der Aminogruppen nicht verändert wird. - Bei der Säurebehandlung der Polymaleonyl-Derivate der 5 genannten Inhibitoren verläuft die Freisetzung der trypsinhemmenden Wirkung (Tab. 3) in allen Fällen parallel zur Freisetzung der Hemmaktivität gegenüber den anderen in Betracht kommenden Enzymen.

Bei den Arginin-Inhibitoren aus Gl. submand. vom Hund, aus Sojabohnen und aus Erdnüssen bleibt dagegen die Plasmin-hemmende Wirkung nach Umsetzung mit Maleinsäureanhydrid voll erhalten (Tab. 4). Bei der Einwirkung des Butandion(2.3)-Reagens auf die Polymaleoyl-Derivate dieser 3 Inhibitoren nimmt ihre Antiplasminaktivität (innerhalb der methodischen Fehlergrenzen) parallel zur Antitrypsinaktivität (Tab. 5 und 6) ab.

Für die Antiplasminwirkung dieser 3 Inhibitoren ist demnach, ebenso wie für die Trypsinhemmung, ein Argininrest zuständig. - Beim Inhibitor aus Eiklar (Ovomucoid) könnte die starke Zunahme der Plasmin-hemmenden Wirkung nach Substitution der Aminogruppen (vgl. Tab. 4) auf die Dissoziation von Polymeren zurückzuführen sein. Die Antiplasminaktivität des nativen Ovomucoid-Präparates ist jedoch auffallend gering und wahrscheinlich nicht an die trypsinhemmende Komponente des Ovomucoid-Inhibitors gebunden.

Die antichymotryptische Aktivität des Inhibitors aus Gl. submand. wird weder durch die Acylierung der Aminogruppen, noch durch die Modifizierung der Arginin-Reste beeinträchtigt: Vor der Reaktion mit Maleinsäureanhydrid hemmt $1 \mathrm{mg}$ 
Tab. 4. Arginin-Inhibitoren. Verbleibende Aktivität nach Umsetzung mit Maleinsäureanhydrid und anschließender Reaktion mit dem Butandion-(2.3)-Reagens.

Die Reaktionsbedingungen sind im Abschnitt Methodik beschrieben. Die Inaktivierungsgeschwindigkeit bei der Umsetzung mit dem Butandion-(2.3)-Reagens geht aus Tab. 5 hervor; vgl. auch Abb. 1.

\begin{tabular}{|c|c|c|c|c|c|c|}
\hline \multirow{3}{*}{ Trypsininhibitor aus } & \multirow{2}{*}{\multicolumn{2}{|c|}{$\begin{array}{c}\text { Spezif. Aktiv. }^{\mathrm{a}} \\
{[\mathrm{IU} / \mathrm{mg}]}\end{array}$}} & \multicolumn{4}{|c|}{ Verbleibende Aktivität in $\%$ bach Umsetzung mit } \\
\hline & & & \multicolumn{2}{|c|}{ Maleinsäureanhydrid } & \multicolumn{2}{|c|}{ Butandion-(2.3)-Reagens } \\
\hline & Trypsin & Plasmin & Trypsin & Plasmin & Trypsin & $\begin{array}{c}\text { Reaktionszeit } \\
\text { [Min.] } \\
\end{array}$ \\
\hline Schafspankreas & 3,3 & & 100 & & 2 & 130 \\
\hline Gl. submand. (Hund) & 1,4 & 0,035 & 100 & 100 & 5 & 133 \\
\hline Meerschw. Samenblasen $I^{c}$ & $3,9 \bigcirc$ & & 32 & & 3 & 210 \\
\hline Sojabohnen & 1,7 & 0,076 & 106 & 99 & 3 & 210 \\
\hline Eiklar (Ovomucoid) & 1,6 & 0,0045 & 97 & 300 & 0,3 & 210 \\
\hline Weizenkeime I & 2,0 & & 100 & & 4 & 132 \\
\hline Roggenkeime I & 2,4 & & 100 & & 3 & 164 \\
\hline Weißbohnen ${ }^{c}$ & 4,5 & & $33(36)^{d}$ & & 34 & 132 \\
\hline Kartoffeln I & 1,4 & & $85(108)^{d}$ & & $<1,0$ & 90 \\
\hline Erdnuß PNT & 6,8 & 0,18 & $106(100)^{d}$ & 100 & $<0,3$ & 90 \\
\hline $\begin{array}{l}\text { Inter- } \alpha \text {-Trypsin-Inhibitor } \\
\text { (Humanserum) }\end{array}$ & 0,37 & & 84 & & $<2,5$ & 90 \\
\hline
\end{tabular}

a Substrat: BAPNA.

c Lysin-Inhibitoren, s. Text.

b Bezogen auf die zur Umsetzung mit Maleinsäureanhydrid bzw. mit dem Butandion-(2.3)-Reagens jeweils eingesetzte Inhibitord Die in Klammern angegebenen Werte wurden bei Ansätzen eraktivität. halten, bei denen die Umsetzung in Gegenwart von $6 \mathrm{M}$ Harnstoff durchgeführt wurde.

Tab. 5. Arginin-Inhibitoren. Abnahme der Antitrypsinaktivität von Polymaleoyl-Derivaten bei der Umsetzung mit Butandion-(2.3)-Reagens (vgl. dazu Tab. 4).

Die Versuchsdurchführung ist im Abschnitt Methodik beschrieben.

\begin{tabular}{|c|c|c|c|c|c|c|c|c|c|c|c|c|c|c|c|}
\hline $\begin{array}{l}\text { Trypsininhibitor } \\
\text { aus }\end{array}$ & $\begin{array}{l}\text { Eingesetzte In- } \\
\text { hibitoraktivität } \\
{[\mathrm{mIU} / \mathrm{m} /]}\end{array}$ & {$[\%]$} & $\begin{array}{l}\text { Verble } \\
\text { [Min.] }\end{array}$ & [\%] & $\begin{array}{l}\text { de Ant } \\
\text { [Min.] }\end{array}$ & $\begin{array}{l}\text { titryp } \\
\text { ange } \\
{[\%]}\end{array}$ & $\begin{array}{l}\text { sinakt } \\
\text { geben } \\
\text { [Min. }\end{array}$ & $\begin{array}{l}\text { tivität } \\
\text { en } \mathrm{Re} \\
\text {.] [\%] }\end{array}$ & $\begin{array}{l}\text { in } \% n \\
\text { aktions } \\
\text { [Min.] }\end{array}$ & $\begin{array}{l}\text { nach } \\
\text { szeit } \\
][\%]\end{array}$ & $\begin{array}{l}\text { der jev } \\
\text { in Mir } \\
\text { [Min. }\end{array}$ & $\begin{array}{l}\text { weils }( \\
\text { n. } \\
\text { ] [\%] }\end{array}$ & $\begin{array}{l}\text { (in Kla } \\
{[\mathrm{Min} .]}\end{array}$ & $\begin{array}{l}\text { amme } \\
{[\%]}\end{array}$ & $\begin{array}{l}\text { ern) } \\
\text { ][Min.] }\end{array}$ \\
\hline Schafspankreas & 1053 & 86 & (3) & & & 37 & (15) & 4 & (39) & 3 & (79) & 2 & (130) & & \\
\hline $\begin{array}{l}\text { Gl. Submand. } \\
\text { (Hund) }\end{array}$ & 500 & & & & & & & 12 & (87) & & & 7 & (133) & & \\
\hline $\begin{array}{l}\text { Meerschw. } \\
\text { Samenblasen I }\end{array}$ & 352 & & & 69 & (9) & & & & & 8 & (101) & & & 3 & (210) \\
\hline Sajabohnen & 1252 & & & 72 & (7) & & & & & 5 & (99) & & & 1 & (210) \\
\hline $\begin{array}{l}\text { Eiklar } \\
\text { (Ovomucoid) }\end{array}$ & 1000 & & & 75 & (8) & & & & & 9 & $(100)$ & & & 0,3 & (210) \\
\hline Weizenkeime I & 653 & & & & & 78 & (17) & 9 & (85) & & & 4 & (132) & & \\
\hline Roggenkeime I & 804 & & & & & & & 15 & (65) & & & 3 & (164) & & \\
\hline Weißbohnen & 503 & & & & & & & 63 & (86) & & & 34 & (132) & 10 & $(960)$ \\
\hline Kartoffeln I & 600 & & & & & & & & & $<1$ & (90) & & & & \\
\hline Erdnuß PNT & 3000 & & & & & & & & & $=0,3$ & $(90)$ & & & & \\
\hline $\begin{array}{l}\text { Inter- } \alpha \text {-Trypsin- } \\
\text { hibitor (Human }\end{array}$ & m) 140 & & & & & & & & & $=2,5$ & (90) & & & & \\
\hline
\end{tabular}


Tab. 6. Arginin-Inhibitoren. Abnahme der Antiplasminaktivität der Polymaleoyl-Derivate bei der, Umsetzung mit dem Butandion-(2.3)-Reagens.

\begin{tabular}{|c|c|c|c|}
\hline \multirow[t]{2}{*}{ Inhibitor aus } & \multicolumn{3}{|c|}{$\begin{array}{c}\text { Verbleibende Antiplasminaktivität in } \% \\
\text { nach der Reaktionszeit von }\end{array}$} \\
\hline & $10 \mathrm{Min}$. & $100 \mathrm{Min}$. & 17 Std. \\
\hline Gl. Submand. & 28 & 8,5 & $<5$ \\
\hline Sojabohnen & 42 & 5 & $<2$ \\
\hline Erdnuß & 32 & 4 & $<2$ \\
\hline
\end{tabular}

dieses Inhibitors die Aktivität von 1,61 mg Chymotrypsin, danach die von 1,54 mg und nach Modifizierung der Guanidinogruppe die von $2,05 \mathrm{mg}$ Chymotrypsin.

Eine weitere Möglichkeit zur selektiven Modifizierung der Guanidinogruppen von Argininresten mit Hilfe von Phenylglyoxal wird in der Diskussion erörtert.

\section{Diskussion}

LASKOWSKI JR. und Mitarb. ${ }^{1-3}$ konnten am Beispiel der Inhibitoren aus Sojabohnen und aus Hühnereiklar (Ovomucoid) erstmals zeigen, daß Proteaseinhibitoren - in Analogie zum aktiven Zentrum der Enzyme - ein sog. reaktives Zentrum besitzen. Bei Inkubation der nativen Inhibitoren mit 1-2 Mol-\% Trypsin in schwach saurer Lösung (pH 3,75) wird bei jedem Inhibitor nur 1 Bindung gespalten; und zwar die Arg-Ile-Bindung beim Inhibitor aus Sojabohnen und die Arg-Ala-Bindung beim Inhibitor aus Hühnereiklar. Die so modifizierten Inhibitoren reagieren bei der Komplexbildung mit Trypsin langsamer als die nativen und verlieren bei der Inkubation mit Carboxypeptidase $B$ infolge der Abspaltung der Argininreste ihre Hemmwirkung.

In Gegenwart äquimolarer Mengen von Trypsin und Inhibitor wird die Bindung im reaktiven Zentrum des modifizierten Inhibitors aus Sojabohnen wieder synthetisiert ${ }^{2}$. Nach neueren Untersuchungen wird durch Einwirkung katalytischer Mengen Trypsin (4-5 Mol-\%) sowohl auf den modifizierten Inhibitor als auch auf den nativen bei $\mathrm{pH} 4,0$ ein Gleichgewichtszustand erreicht, in dem $86 \%$ des Inhibitors in modifizierter und $14 \%$ in nativer Form vorliegen ${ }^{25}$. Wird das nach Abspaltung des Argininrestes aus dem modifizierten Inhibitor erhaltene inaktive Des-Arg-Protein in Gegenwart ge-

25 C. W. Niekamp, H. F. Hixson JR. u. M. Laskowski JR., Biochemistry [Washington] 8, 16 [1969].
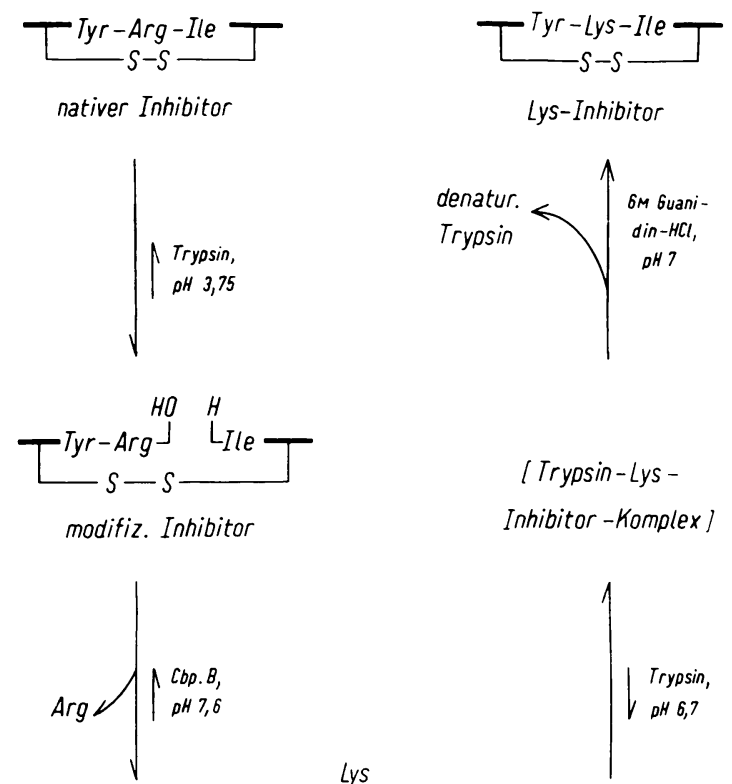

[Trypsin-Lys -
Inhibitor-Komplex]

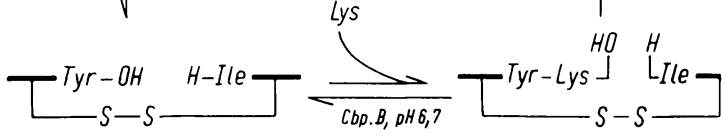

inaktiver Des-Arg- "Inhibitor"

modifiz. Lys-Inhibitor

Abb. 2. Schematische Darstellung der Modifizierungsreaktionen beim Inhibitor aus Sojabohnen nach LASKOWSKI JR. und Mitarb. ${ }^{26}$ (vgl. Text).

eigneter Mengen von Lysin, Carboxypeptidase B und Trypsin inkubiert, so erhält man einen neuen Inhibitor, der statt des Argininrestes einen Lysinrest enthält ${ }^{26}$. Die Lys-Ile-Bindung im reaktiven Zentrum des Lysin-Inhibitors läßt sich durch Trypsineinwirkung wieder spalten (langsamer als beim nativen Inhibitor), so daß auch der modifizierte Lysin-Inhibitor darstellbar ist ${ }^{26}$ (vgl. Abb. 2). Aus diesen sehr elegant durchgeführten Untersuchungen geht hervor, daß im reaktiven Zentrum des Inhibitors ein Aminosäurerest vorhanden sein muß, für den das zu inkubierende Trypsin ,,von Natur aus“ eine hohe Affinität besitzt (Arginin oder Lysin). Für die geringe Dissoziation des Trypsin-InhibitorKomplexes dürften jedoch noch andere Gruppen, die zusätzliche Bindungskräfte beisteuern, verantwortlich $\operatorname{sein}^{3}$.

HAYNES und FeENEY ${ }^{27}$ blockierten die freien $x$ -

26 R. B. Sealock u. M. Laskowski JR., Biochemistry [Washington], im Druck.

27 R. HAynes u. R. E. Feeney, Biochim. biophysica Acta [Amsterdam] 159, 209 [1968]. 
Aminogruppen im reaktiven Zentrum der modifizierten Inhibitoren durch Umsetzung mit Trinitrobenzolsulfonsäure: Der Inhibitor aus Sojabohnen verliert dabei rasch, der Inhibitor aus Hühnereiklar nur langsam seine Hemmwirkung. Erfolgte die Umsetzung nach vorheriger Amidinierung der modifizierten Hemmstoffe(Acylierung der $\alpha$-Aminogruppe mit einem Acetimidoylrest), so trat kein Aktivitätsverlust auf. Dies, sowie auch unsere Ergebnisse (s. u.) sprechen dafür, daß geringere chemische Veränderungen der $\alpha$-Aminogruppen ohne Einfluß auf die Hemmwirkung modifizierter Inhibitoren sind.

Außer bei den zwei genannten Arginin-Inhibitoren besitzen wir auch Einblick in den Bau des reaktiven Zentrums des polyvalenten Proteaseinhibitors aus Rinderorganen (Kallikrein-Inaktivator, KunITZInhibitor): Die Hemmwirkung dieses Inhibitors gegenüber Trypsin ist aufgehoben nach Acylierung (Acetylierung, Succinylierung und Reaktion mit $N$-Carboxy-alanin-anhydrid*) und Desaminierung aller freien Aminogruppen, nicht aber nach deren Guanidierung 28, 29. Bei der Umsetzung des Inhibitors mit $\mathrm{N}$-Carboxy-alanin-anhydrid reagieren die $4 \varepsilon$-Aminogruppen der Lysinreste interessanterweise mit gleicher Geschwindigkeit, d. h. gleichberechtigt, während die $N$-terminale $\alpha$-Aminogruppe bevorzugt acyliert wird ${ }^{29}$. Setzt man dagegen den Trypsin-Inhibitor-Komplex der Einwirkung von überschüssigem $N$-Carboxy-alanin-anhydrid aus, so wird nur eine einzige Aminogruppe des Inhibitors nicht acyliert, und zwar die $\varepsilon$-Aminogruppe des Lysinrestes in Stellung 15 der Peptidsequenz ${ }^{28}$. ChAUVET und ACHER folgerten daraus, $\mathrm{da} B$ der Lysin-15-Rest das reaktive Zentrum des Inhibitors darstellt.

Untersuchungen im Arbeitskreis von LASKOwSKI SEN. bestätigten diese Annahmen in eindrucksvoller Weise: Die Disulfidbrücke zwischen den CysResten 14 und 38 läßt sich mit Natriumborhydrid selektiv reduzieren, ohne daß die Hemmwirkung des Inhibitors gegenüber Trypsin abnimmt ${ }^{30}$. Werden aber die 2 neu entstandenen Sulf hydrylgruppen, von denen sich eine in unmittelbarer Nachbarschaft des Lysin-15-Restes befindet, mit Sulfhydrylreagenzien substituiert, so entstehen Inhibitorderivate mit unterschiedlichen Eigenschaften ${ }^{30,31}$ (Tab. 7).

* 4-Methyl-oxazolidindion-(2.5).

28 J. Chauvet u. R. Acher, J. biol. Chemistry 242, 4274 [1967]; s. dort sowie bei 1. c. ${ }^{30}$ weitere Literatur.

29 R. Acher, J. Chauvet, R. Arnon u. M. Sela, Europ. J. Biochem. [Berlin] 3, 476 [1968].
Tab. 7. Eigenschaften von Inhibitorderivaten, die durch Substitution der beiden - durch Reduktion freigelegten - Sulfhydrylgruppen mit verschiedenen Reagenzien dargestellt wurden (nach KRESS, WILSON und LASKOwSKI SEN. ${ }^{30,31}$.

\begin{tabular}{|c|c|c|c|c|}
\hline Inhibitorderivat & Hemm & ung von & verdaub & ar durch \\
\hline (Cys-14, Cys-38) & Trypsin & $\begin{array}{l}\text { Chymo- } \\
\text { trypsin }\end{array}$ & Trypsin & $\begin{array}{l}\text { Chymo- } \\
\text { trypsin }\end{array}$ \\
\hline$-\mathrm{CH}_{2} \mathrm{CO}_{2}{ }^{\ominus}$ & - & - & + & + \\
\hline$-\mathrm{CH}_{2} \mathrm{CONH}_{2}$ & + & - & + & \\
\hline$-\left[\mathrm{CH}_{2}\right]_{2} \mathrm{NH}_{2}$ & + & - & + & + \\
\hline$S$ & + & $(+)$ & - & - \\
\hline
\end{tabular}

Daraus ist ersichtlich, daß ein negativ geladener Substituent in unmittelbarer Nachbarschaft des Lysin-15-Restes die Hemmwirkung des Inhibitors aufhebt - evt. bildet sich eine zwitterionische Bindung zwischen der Carboxylatgruppe und der $\varepsilon$-Aminogruppe - nicht dagegen ein annähernd neutraler oder positiv geladener Rest. Bei Inkubation des carbamoylmethylierten Inhibitors mit Trypsin wird der Inhibitor teilweise durch Spaltung der Lys $^{15}$-Ala ${ }^{16}$-Bindung modifiziert, dabei geht allerdings seine Hemmwirkung verloren ${ }^{32}$. Das reaktive Zentrum des polyvalenten Proteaseinhibitors stellt demnach der Lysinrest in Stellung 15 dar. Modifikationen am Lysinrest selbst (Lysin $\rightarrow$ Homoarginin) oder in dessen unmittelbarer Umgebung führen offensichtlich nur dann nicht zum Verlust der Hemmwirkung, wenn die positive Ladung und damit auch die Spezifität dieses Restes für die Reaktion mit Trypsin erhalten bleibt. Auffallend ist dagegen der Verlust der Hemmwirkung gegenüber Chymotrypsin bei 3 der in Tab. 7 angeführten trypsinaktiven Inhibitorderivate.

HAYNES, FEENEY und Mitarbeiter modifizierten bei einer Reihe von Inhibitoren spezifisch die Aminogruppen (mit Trinitrobenzolsulfonsäure) und die Guanidinogruppen (mit Cyclohexandion-(1.2)) um einen Einblick in die Art des reaktiven Zentrums zu erhalten; sie fanden, daß durch die selektive Blok-

30 L. F. Kress u. M. LASKowski SEN., J. biol. Chemistry 242, 4925 [1967].

${ }^{31}$ L. F. KRESS, K. A. Wilson u. M. LASKowSKi SEN., J. biol. Chemistry 243, 1758 [1968].

32 L. F. KResS u. M. Laskowski SEN., J. biol. Chemistry 243, 3548 [1968]. 
kierung der Aminogruppen ( $\varepsilon$ und $\alpha$ ) die Hemmwirkung der Lysin-Inhibitoren (Inhibitor aus Limabohnen und aus Rindercolostrum, Inhibitoren aus Eiklar von Kasuar, Ente, Pinguin und Truthahn) gegenüber Trypsin verlorengeht, während die Hemmaktivität von Arginin-Inhibitoren (Inhibitor aus Sojabohnen und Hühnereiklar) unverändert erhalten bleibt ${ }^{33,34}$. Umgekehrt sind die genannten Arginin-Inhibitoren nach Reaktion mit Cyclohexandion-(1.2) $\mathrm{zu} 80 \%$ inaktiviert, die LysinInhibitoren dagegen nur zu $0-33 \% 35$.

HAYNES und FeENEY ${ }^{36}$ untersuchten auch den Einfluß der Guanidinierung (Umwandlung von Lysin in Homoarginin) und Amidinierung (Umwandlung von Lysin in $N^{\varepsilon}$-Acetimidoyl-lysin) auf die Hemmwirkung von Lysin-Inhibitoren gegenüber Trypsin: Die guanidinierten Inhibitoren (Inhibitor aus Limabohnen und Eiklar von Ente, Truthahn und Kasuar) sind zwar noch aktiv, die Dissoziationskonstanten der Komplexe mit Trypsin sind aber, verglichen mit den nativen Lysin-Inhibitoren, deutlich erhöht. Noch höhere Dissoziationskonstanten besitzen die Trypsin-Inhibitor-Komplexe mit den amidinierten Inhibitoren. Der Ersatz von Lysin im reaktiven Zentrum der Inhibitoren durch Homoarginin oder $N^{\varepsilon}$-Acetimidoyl-lysin, also durch Reste, die zum aktiven Zentrum des Trypsins eine geringere Affinität besitzen als Lysin*, führt demnach zu einer entsprechenden Abschwächung der Hemmwirkung. Diese Befunde und die der Arbeitsgruppen von LASKOWSKI SEN. und JR. sprechen für die Annahme, $\mathrm{da} B$ zumindest für die Trypsinhemmung nicht bislang „unbekannte“ Kräfte verantwortlich sind: Der Inhibitor stellt in erster Linie ein Substrat für Trypsin dar, wobei allerdings der entstehende Michaelis-Menten-Komplex eine ungewöhnliche

* Poly-L-homoarginin, ein Hemmstoff für Trypsin in Gegenwart des Substrates BAPNA $\left(K_{i}: 10^{-5}-10^{-6} \mathrm{M}\right)$ wird z. B. durch Trypsin etwa $200 \mathrm{mal}$ so langsam hydrolysiert wie Poly-L-lysin; L-Homoarginin hemmt Trypsin ebenfalls schwach (M. RigBi u. L. ElbAZ, VI. FEBSMeeting, Abstracts, p. 730 [1969]); vgl. auch M. MARESGuIA, Archiv. Biochem. Biophys. 127, 317 [1968].

33 R. Haynes u. R. E. Feeney, J. biol. Chemistry 242, 5378 [1967].

34 R. Haynes, D. T. Osuga u. R. E. Feeney, Biochemistry [Washington] 6, 541 [1967].

35 W.-H. Liu, G. Feinstein, D. T. Osuga, R. Haynes u. R. E. FEeney, Biochemistry [Washington] 7, 2886 [1968].

${ }^{36}$ R. HAYNES u. R. E. FeENEy, Biochemistry [Washington] 7, 2879 [1968].
Stabilität aufweist. Dabei sind alle Übergangsmöglichkeiten denkbar: Von dem nur durch Nebenvalenzkräfte zwischen den reaktiven Zentren von Inhibitor und Enzym stabilisierten Komplex über die Ausbildung einer kovalenten Bindung zwischen dem Serinrest des Trypsins und dem Lysin- bzw. Argininrest des Inhibitors bis zur ,extrem“ langsam spaltbaren Bindung im reaktiven Inhibitorzentrum.

Erwartungsgemäß werden die genannten guanidinierten Lysin-Inhibitoren durch Einwirkung von Cyclohexandion-(1.2) völlig inaktiviert, während sie bei Behandlung mit Trinitrobenzolsulfonsäure ihre Hemmwirkung beibehalten ${ }^{36}$. Andererseits ist sowohl die Guanidinierung als auch die Amidinierung ohne Einfluß auf die Hemmwirkung des Arginin-Inhibitors aus Hühnereiklar ${ }^{36}$.

Die Ergebnisse unserer Untersuchungen lassen den Schluß zu, daß die genannten Vorstellungen über das reaktive Zentrum von Proteaseinhibitoren allgemeine Gültigkeit besitzen: Die in Tab. 2 angegebenen Lysin-Inhibitoren sind nach Substitution der Aminogruppen mit den negativ geladenen Maleoylresten nahezu vollständig inaktiviert, während die Aktivität der Arginin-Inhibitoren (Tab. 4) davon nicht oder nur sehr wenig beeinflußt wird. Die polymaleoylierten Arginin-Inhibitoren (Tab. 4) werden andererseits durch das nach erfolgter Blockierung der Aminogruppen nun spezifisch die Guanidinogruppen der Argininreste modifizierende Butandion-(2.3)-Reagens relativ rasch und nahezu vollständig inaktiviert (Tab. 4 und 5). Die bei direkter Verwendung von Cyclohexandion-(1.2) auftretenden Nebenreaktionen ${ }^{36}$ (mit den Aminogruppen $^{9}$ ) werden dadurch vermieden.

Die säurestabilen Lysin-Inhibitoren lassen sich aus ihren Polymaleoyl-Derivaten wieder vollständig reaktivieren (Tab. 3). Um säurelabile Lysin-Inhibitoren wieder reaktivieren zu können, empfiehlt es sich, die Blockierung der Aminogruppen mit Hilfe von Tetrafluorbernsteinsäureanhydrid ${ }^{38}$ bzw. von Citraconsäureanhydrid ${ }^{37}$ vorzunehmen; die Deacylierung kann hier bereits bei sehr milden Bedingungen (pH 9,5 und $0^{\circ} \mathrm{C}^{38}$ bzw. $\mathrm{pH} 3,5$ und $20^{\circ} \mathrm{C}^{37}$ ) durchgeführt werden.

Die polyvalenten Lysin-Inhibitoren in Tab. 2 verlieren bei der Acylierung der Aminogruppen nicht

37 H. B. F. Dixon u. R. N. Perham, Biochem. J. 109, 312 [1968].

38 G. Braunitzer, K. Beyreuther, H. Fujiki u. B. Schrank, diese Z. 349, 265 [1968]. 
nur ihre Hemmwirkung gegenüber Trypsin, sondern auch gegenüber Plasmin, Chymotrypsin und Kallikrein; nur beim Inhibitor aus Limabohnen bleibt die antichymotryptische Wirkung erhalten. Diese und weitere Versuchsergebnisse ${ }^{39}$ sprechen dafür, daß für die Antiplasminwirkung und, soweit zutreffend, auch für die Antikallikreinwirkung dieser Inhibitoren der gleiche Lysinrest das reaktive Zentrum darstellt. Beim Inhibitor aus Limabohnen ist die antichymotryptische Wirkung evt. an einen aromatischen Aminosäurerest gebunden. Noch keine plausible Erklärung haben wir für das Verschwinden der antichymotryptischen Wirkung nach Maleoylierung der polyvalenten Inhibitoren aus Lunge von Rind und Schaf.

Die drei Inhibitoren aus Gl. submand., Sojabohnen und Erdnüssen besitzen nach der Maleoylierung noch ihre volle Antiplasminwirkung (Tab. 4). Sie verlieren diese erst - analog zur Antitrypsinwirkung - bei der Umsetzung mit dem Butandion(2.3)-Reagens. Für beide Wirkungen dürfte deshalb bei jedem der 3 genannten Inhibitoren dasselbe reaktive Zentrum, nämlich ein Argininrest, zuständig sein. - Die starke Steigerung der Antiplasminwirkung beim Inhibitor aus Hühnereiklar ist evt. auf die Dissoziation von Assoziaten zurückzuführen ${ }^{40,41}$.

Die von Hochstrasser und Mitarb. (vgl. Tab. 8) über wasserunlösliche Trypsinharze isolierten Inhibitoren aus Pflanzenkeimen fallen teilweise bis überwiegend in modifizierter Form an: Die Spaltung der Peptidbindung im reaktiven Zentrum dürfte dabei während der Isolierungsprozedur erfolgen, bei der die Inhibitoren längere Zeit bei $\mathrm{pH}$-Werten zwischen 2 und 8 mit Trypsin im Komplex verbunden sind ${ }^{21-24,42}$. Die Maleoylierung der $\alpha$-Aminogruppen des Leucin- bzw. Alaninrestes im reaktiven Zentrum (Tab. 8) hat offensichtlich keinen Einfluß auf die Hemmaktivität dieser Inhibitoren (Tab. 4). Die gegensätzlichen Befunde von HAYNES und FEENEY ${ }^{27}$ bei den modifizierten Inhibitoren aus Sojabohnen und Hühnereiklar sind wahrscheinlich auf die dort verwendete relativ raumfüllende Trinitrobenzolsulfonsäure als blockierendes Agens zu-

\footnotetext{
${ }^{39}$ H. Fritz, H. Schult, K.-H. Oppitz, M. Gebhardt, R. Meister u. E. Werle, in Vorbereitung.

40 D. B. S. Millar, G. E. Willick, R. F. Steiner u. V. Frattali, J. biol. Chemistry 244, 281 [1969].

$41 \mathrm{~K}$. Hochstrasser, K. IllchmanN u. E. Werle, diese Z. 350, 655 [1969].

42 K. Hochstrasser, M. Muss u. E. Werle, diese Z. 348, 1337 [1967].
}

Tab. 8. Reaktive Zentren von Proteaseinhibitoren.

Es sind nur Inhibitoren aufgeführt, bei denen das reaktive Zentrum durch unabhängige Untersuchungen der angegebenen Autoren aufgeklärt wurde. Weitere Lysin- bzw. Arginin-Inhibitoren sind in den Tab. 2 und 4 enthalten.

\begin{tabular}{lll}
\hline \multirow{2}{*}{$\begin{array}{l}\text { Trypsininhibitor } \\
\text { aus }\end{array}$} & \multicolumn{2}{l}{ Aminosäure bzw.Sequenz Autoren } \\
im Hemmzentrum & Lit.cit. \\
\hline Sojabohnen & -Arg-Ile- & $1-3,25$ \\
Hühnereiklar & -Arg-Ala- & 3 \\
Maiskeimen & -Arg-Leu- & 42 \\
Roggenkeimen & -Arg-Ala- & 21 \\
Weizenkeimen & -Arg-Ala- & 21 \\
Erdnuß & -Arg-Ala- & 24 \\
Rinderpankreas & -Arg-Ile- & 43 \\
Schafspankreas & -Arg-(Ile)-a & 44 \\
Schweinepankreas & -Lys-Ile- & 45,46 \\
Rinderlunge & & $28,30-32$ \\
\multicolumn{2}{c}{ (Rinderpankreas) } & -Lys-Ala- \\
Schafslunge & -Lys-(Ala)-a & 47 \\
\hline
\end{tabular}

a Aufgrund identischer Aminosäurezusammensetzungen angenommen.

rückzuführen, wodurch die Hemmwirkung aus sterischen Gründen abgeschwächt bzw. aufgehoben wird.

Beim Inhibitor aus Weißbohnen wird die nach der Acylierung verbleibende Hemmaktivität (Tab. 4 und 2) weder durch Zugabe von mehr Maleinsäureanhydrid zum Reaktionsansatz noch durch die Gegenwart von 6M Harnstoff bei der Acylierung weiter vermindert, obwohl die Kinetik der Inaktivierung mit dem Butandion-(2.3)-Reagens (Abb. 1) dafür spricht ${ }^{9}$, da $\beta$ eine Aminogruppe für diese Restaktivität verantwortlich ist. Evt. besitzt dieser Inhibitor ein zweites reaktives Lysin-Zentrum mit geringerer Affinität zum Trypsin, dessen Lysinrest aus sterischen Gründen nicht acyliert wird. Beim Inhibitor I aus Meerschweinchen-Samenblasen wäre das Vorhandensein eines zweiten, „schwächeren“

43 M. Rigbi u. L. J. Greene, J. biol. Chemistry 243, 5457 [1968].

44 K. Hochstrasser, W. Schramm, H. Fritz, S. SchWARZ u. E. Werle, diese Z. 350, 893 [1969].

45 H. TSCHESCHE, diese Z. 348, 1653 [1967]; H. TSCHESCHE U. H. KLEIN, diese Z. 349, 1645 [1968]; H. TsCHESCHE, persönl. Mitteil.

46 L. J. Greene, persönl. Mitteil.

47 H. Fritz, K. Hochstrasser, W. Schramm u. E. WERLE, in Vorbereit. 
Tab. 9. Modifizierung der Argininreste von Inhibitoren mit Phenylglyoxal.

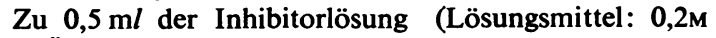
$N$-Äthyl-morpholin/Essigsäure, pH 8,0) wurden $2 \mathrm{mg}$ Phenylglyoxal (beim Inhibitor aus Sojabohne: $5 \mathrm{mg}$ !)

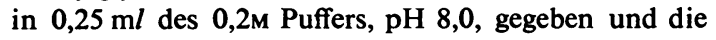
Reaktionsmischung bei Zimmertemperatur inkubiert. $\mathrm{Zu}$ den angegebenen Zeitpunkten wurden Proben entnommen und auf ihren Inhibitorgehalt getestet.

\begin{tabular}{lccccr}
\hline Inhibitor aus & $\begin{array}{c}\text { Inhibitor- } \\
\text { aktivität der } \\
\text { Ausgangs- } \\
\text { lösung }\end{array}$ & \multicolumn{4}{c}{$\begin{array}{c}\text { Verbleibende Inhibitor- } \\
\text { aktivität in \% nach der }\end{array}$} \\
& $\begin{array}{c}\text { zeit in Min. } \\
\text { [mIU/ml] }\end{array}$ & 15 & 60 & 70 & 200 \\
\hline Schafspankreas & 3800 & 84 & & 25 & 5 \\
Sojabohnen & 7500 & $24^{\mathrm{a}}$ & $16^{\mathrm{a}}$ & & \\
Roggenkeimen I & 2470 & 75 & & 18 & 4 \\
Rinderlunge & 5130 & 95 & & 95 & 72 \\
\hline
\end{tabular}

a Höhere Konzentration an Phenylglyoxal, s. Text.

Arginin-Zentrums denkbar. Wir können jedoch bei beiden Präparaten das Vorliegen von Inhibitorgemischen noch nicht mit Sicherheit ausschlieBen.

Zur reversiblen Blockierung von Argininresten eignet sich nach neueren Untersuchungen von TAKAHASHI besonders Phenylglyoxal ${ }^{48}$. Dieses Reagens hat den Vorteil, daß damit im Gegensatz zu Cyclohexandion-(1.2) ${ }^{49}$ und Butandion- $(2.3)^{9}$ die Modifizierung freier Aminogruppen sehr viel langsamer erfolgt als die der Guanidinogruppen. Dies geht auch aus den Angaben in Tab. 9 hervor: Die nativen Arginin-Inhibitoren aus Schafspankreas, Sojabohnen und Roggenkeimen I werden durch Phenylglyoxal sehr viel rascher inaktiviert als der native Lysin-Inhibitor aus Rinderlunge. Die so modifizierten Argininreste lassen sich unter relativ schonenden Bedingungen (48stdg. Inkubation bei $37^{\circ} \mathrm{C}$ und $\mathrm{pH} 7,0^{48}$ ) wieder regenerieren; die in der Tab. 9 angegebenen Arginin-Inhibitoren gewinnen dabei ihre Hemmaktivität teilweise wieder zurück.

Anm. b. d. Korr. (22. 7. 1969): Für weitere Unter suchungen, die sich mit dem reaktiven Zentrum von Inhibitoren befassen, s. V. Frattali u. R. F. STEINER, Biochem. biophysic. Res. Commun. 34, 480 [1969] sowie G. Birk, A. Gertler u. S. Khalef, Biochim, biophysica Acta [Amsterdam] 147, 402 [1967].

48 K. Takahashi, J. biol. Chemistry 243, 6171 [1968].

49 K. Toi, E. Bynum, E. Norris u. H. A. Itano, J. biol. Chemistry 242, 1036 [1967]. 